\title{
ORIGINAL
}

\section{Evaluation of the use of intraoperative real-time virtual sonography with sonazoid enhancement for detecting small liver metastatic lesions after chemotherapy in hepatic resection}

\author{
Kenichiro Araki, M.D., Ph.D., Norifumi Harimoto, M.D., Ph.D, Ryo Muranushi, M.D., Kouki Hoshino, M.D., \\ Kei Hagiwara, M.D., Takahiro Yamanaka, M.D., Norihiro Ishii, M.D., Ph.D, Mariko Tsukagoshi, M.D., Ph.D, \\ Takamichi Igarashi, M.D., Ph.D, Akira Watanabe, M.D., Ph.D, Norio Kubo, M.D., Ph.D., and Ken Shirabe, M.D., Ph.D. \\ Division of Hepatobiliary and Pancreatic Surgery, Department of General Surgical Science, Gunma University Graduate School of Medicine, \\ Gunma, Japan
}

\begin{abstract}
Background: Systemic chemotherapy can drastically downsize metastatic liver tumors and these small liver lesions could sometimes be difficult for surgeons to detect during hepatectomy. We assessed the usefulness of intraoperative real-time virtual sonography (RVS) with contrast-enhanced ultrasonography (CEUS) using 'Sonazoid' contrast agent (RVS-CEUS). Methods : We performed the intraoperative RVS-CEUS technique on 10 tumor lesions in six cases, which were scheduled for hepatic resection of $<10 \mathrm{~mm}$ in diameter in our liver metastases series. These lesions were preoperatively diagnosed by contrast enhanced-computed tomography (CE-CT) or Gadolinium-ethoxybenzyl-diethylenetriamine pentaacetic acid (Gd-EOB-DTPA)-enhanced magnetic resonance imaging (EOB-MRI). We assessed the detectability of a tumor with RVS-CEUS during surgery and compared it with that of preoperative CE-CT or EOB-MRI. Results : Detectability of RVS-CEUS for 10 small lesions was $90 \%(n=9 / 10)$ and that of other preoperative modalities were $50 \%(n=5 / 10$, CE-CT) and $100 \%(n=10 / 10$, EOB-MRI). Minimum tumor size detected was $3.0 \mathrm{~mm}$ in diameter, and maximum depth of detection with RVSCEUS was $43.5 \mathrm{~mm}$; these results could be an advantage when compared with other intraoperative diagnostic modalities. Conclusion : Intraoperative RVS-CEUS was useful for detecting small metastatic liver lesions after chemotherapy and could be an effective intraoperative diagnostic technique for hepatic resection of a size $<10$ mm. J. Med. Invest. $66: 319-323$, August, 2019
\end{abstract}

Keywords : real-time virtual sonography, small liver metastatic lesion, sonazoid enhancement, hepatectomy, chemotherapy

\section{INTRODUCTION}

Since considerable advancements have been made in systemic chemotherapies for the treatment of colorectal cancer, aggressive surgical resection combined with systemic chemotherapy for colorectal liver metastases (CRLM) has become a standard treatment (1). These chemotherapies can reduce the tumor size of CRLM, allowing radical resection unless tumors were initially unresectable $(2,3)$. Several reports described that the incidence of 'disappearing' liver metastases (DLM) in patients receiving preoperative systemic chemotherapy ranged from 7-37\% (4-6). The phenomenon of disappearing small liver metastases seems to be a radiological rather than an actual biological occurrence, because the rates of macroscopic and microscopic residual disease are high and the local recurrence rates are high as well (7). Therefore, surgeons must detect these downsized and small lesions of metastatic liver tumors accurately during surgery for radical resection.

Preoperative imaging for the diagnosis of hepatic lesions has been extremely developed with the advent of contrast-enhanced ultrasonography (CEUS) and Gadolinium-ethoxybenzyl-diethylenetriamine pentaacetic acid-enhanced magnetic resonance

Received for publication May 13, 2019 ; accepted July 12, 2019.

Address correspondence and reprint requests to Kenichiro Araki, M.D., Ph.D. Division of Hepatobiliary and Pancreatic Surgery, Department of General Surgical Science, Gunma University Graduate School of Medicine, 3-39-22 Showa-machi, Maebashi, Gunma 3718511, Japan and Fax : +81-27-220-8230.
(MR) images (EOB-MRI). Ultrasoundcontrast microbubbles (MBs), such as Sonazoid MBs, make it possible to render a specific phase caused by phagocytosis of Sonazoid MBs by Kuppfer cells as late vascular phase (8). Gd-EOB-DTPA, which is taken up by hepatocytes and excreted in the bile, can make it easy to improve the detection and characterization of small hepatic tumors (9-11). Using these new modalities, hepatic lesions of a few millimeters in size could be detected. However, these small hepatic lesions are often difficult to detect using intraoperative ultrasound (IOUS) during surgery, because of the dissociation between images and intraoperative real-vision of the liver.

Real-time virtual sonography (RVS), also known as real-time fusion imaging (Fusion Imaging), is an innovative technique that can visualize both scanned ultrasound images and reconstruction images of computed tomography (CT) and/or magnetic resonance (MR) on the same screen of the ultrasound system. This imaging technique has already been applied for navigation, to guide needle puncture in radiofrequency ablation therapy (12), prostate biopsy (13), and identification of breast lesions (14). From these facts, we applied the RVS system and CEUS technology (RVS-CEUS) for intraoperative use in hepatic resection. In this article, we report the significance of RVS-CEUS in detecting small liver lesions as a novel technique in hepatic resection.

\section{METHODS}

Patients and Inclusion criteria

From June 2016 to December 2018, the RVS-CEUS method 
was indicated in six cases of hepatic resection, which included 10 liver lesions, at the Gunma University Hospital. The patients were selected as follows : malignant hepatic lesions scheduled for liver resection; small lesion less than 10 millimeters; both dynamic CT and EOB-MRI performed preoperatively ; lesion expected to be very difficult to detect intraoperatively by surgeons ; and anticipated curative resection of the liver lesions by hepatic resection. The patient characteristics are shown in Table 1. Our institutional ethics committee approved the study protocol (Approval No. : 2018-247).

\section{Preoperative imaging analysis}

Routinely, we reconstruct virtual reality images from CT images using SYNAPSE VINCENT software (Fujifilm medical, Tokyo, Japan) for preoperative three-dimensional (3D) simulation of hepatic resection. To prepare for using the intraoperative RVS method, preoperative CT or MRI images and digital imaging and communication in medicine (DICOM) data were uploaded to the RVS ultrasound system before surgery, and we reconstructed real-time fusion imaging on the monitor of the RVS ultrasound system during surgery (the operative procedure is described below).

\section{Procedural steps of RVS technique and Sonazoid enhancement}

For the RVS technique, we used the APLIO i800 system (Toshiba Medical Systems Corp., Otawara, Japan). The procedural steps of IO-RVS were as follows : RVS ultrasound system was placed on the left side of the patient's head. The electromagnetic transmitter/receiver, which is connected to the RVS ultrasound system, was placed on the left side of the patient's neck. The convex type of IOUS probe was attached to the sterilized magnetic sensor. This electromagnetic transmitter and the sensor detect the spatial position and direction of the ultrasound probe using a magnetic field. Then, the spatial positions of both the ultrasound and CT/MR images were adjusted appropriately. The axial plane of CT/MR images was screened with landmarks of the liver such as the portal and hepatic veins for adjustment.
Next, the probe was placed on the liver surface to adjust fusion visualization between the ultrasound image and CT/MR images. When the ultrasound probe was moved on surface of the liver, real-time multiplanar reconstruction (MPR) images of CT/MR could be visualized intraoperatively in the RVS system (Fig. 1A).

The procedural steps of Sonazoid enhancement were as follows : after preparation of the RVS system, the ultrasound probe was put around the target lesion on the surface of the liver with reference to preoperative $3 \mathrm{D}$ simulation images. The $0.5 \mathrm{ml}$ solution of Sonazoid MBs was injected into the patients intravenously, and then we observed and detected target lesions with the ultrasound system. Metastatic tumors are almost visualized as low echoic lesions in late vascular phase, which is after fiveto-ten minutes after injection of Sonazoid MBs. The APLIO i800 system is able to visualize both the Sonazoid-enhanced ultrasound image and the RVS image on the same monitor (Fig. 1B).

\section{Statistical analysis}

Continuous data are presented as mean (standard deviation [SD]) and median (range). Differences between the two cohorts were assessed using Fisher's exact test and the Mann-Whitney $\mathrm{U}$ test, as appropriate. All statistical analyses were performed using SPSS Statistics software version 24.0 (IBM SPSS Inc., Chicago, IL). $P<0.05$ was considered statistically significant.

\section{RESULTS}

The results of the study are summarized in Table 1 . All of the target lesions were from six cases and 10 lesions $(n=10)$ of metastatic liver tumors, which were derived from patients with colorectal cancer $(n=5)$, and adenosquamous carcinoma of the uterus $(n=1)$. The regimens of chemotherapy were as follows : XELOX base $(n=3)$, FOLFOX base Bevacizumab $(n=1)$, and paclitaxel + CDDP $(n=1)$. Macroscopic pathological findings of the liver were revealed in three cases with a normal liver, two with a steatotic liver, and one with a blue liver. Microscopic

Table 1. Summary of case series performed RVS using Sonazoid enhancement

\begin{tabular}{|c|c|c|c|c|c|c|c|c|c|c|c|c|c|}
\hline $\begin{array}{c}\text { Case } \\
\text { No. }\end{array}$ & $\begin{array}{c}\text { Tumor } \\
\text { No. }\end{array}$ & Age & Sex & Diagnosis & $\begin{array}{c}\text { Segment } \\
\text { of lesion }\end{array}$ & $\begin{array}{c}\text { Tumor } \\
\text { diameter }\end{array}$ & $\begin{array}{c}\text { Depth } \\
\text { of tumor }\end{array}$ & $\begin{array}{c}\text { Regimen of } \\
\text { chemotherapy }\end{array}$ & $\begin{array}{c}\text { macroscopic } \\
\text { change of } \\
\text { liver }\end{array}$ & $\begin{array}{c}\text { microscopic } \\
\text { change of } \\
\text { liver }\end{array}$ & $\begin{array}{c}\text { Detection } \\
\text { by CE- } \\
\text { CT }\end{array}$ & $\begin{array}{c}\text { Detection } \\
\text { of EOB- } \\
\text { MRI }\end{array}$ & $\begin{array}{c}\text { Detection } \\
\text { of RVS- } \\
\text { CEUS }\end{array}$ \\
\hline 1 & 1 & 62 & $\mathrm{M}$ & CRLM & 7 & 5.0 & 43.5 & FOLFOX+Bev & normal & $\begin{array}{c}\text { Inflammatory } \\
\text { cell } \\
\text { infiltration }\end{array}$ & + & + \\
\hline 2 & 2 & 54 & $\mathrm{~F}$ & CRLM & 6 & 4.5 & 0 & XELOX & $\begin{array}{c}\text { steatotic } \\
\text { liver }\end{array}$ & $\begin{array}{c}\text { large fat } \\
\text { droplet }\end{array}$ & - & + & + \\
\hline & 3 & & & & 6 & 4.0 & 7.3 & & & & - & + & + \\
\hline & 4 & & & & 8 & 4.6 & 11.6 & & & & + & + & + \\
\hline 3 & 5 & 52 & $\mathrm{M}$ & CRLM & 5 & 4.8 & 20.0 & XELOX+Bev & normal & normal & + & + & + \\
\hline 4 & 6 & 49 & $\mathrm{~F}$ & $\begin{array}{c}\text { adenosquamous } \\
\text { carcinoma of }\end{array}$ & 5 & 5.2 & 13.4 & $\begin{array}{c}\text { Paclitaxel } \\
\text { +CDDP }\end{array}$ & $\begin{array}{c}\text { steatotic } \\
\text { liver }\end{array}$ & large fat & droplet & - & + \\
\hline
\end{tabular}

RVS, real-time virtual sonography ; CE-CT, contrast-enhanced-computed tomography ; EOB-MRI, gadolinium-ethoxybenzyl-diethylene-triaminepentaacetic acid-enhanced magnetic resonance image; RVS-CEUS, contrast-enhanced ultrasonography with real-time virtual sonography ; CRLM, Colorectal liver metastases ; Bev, Bevacizumab 

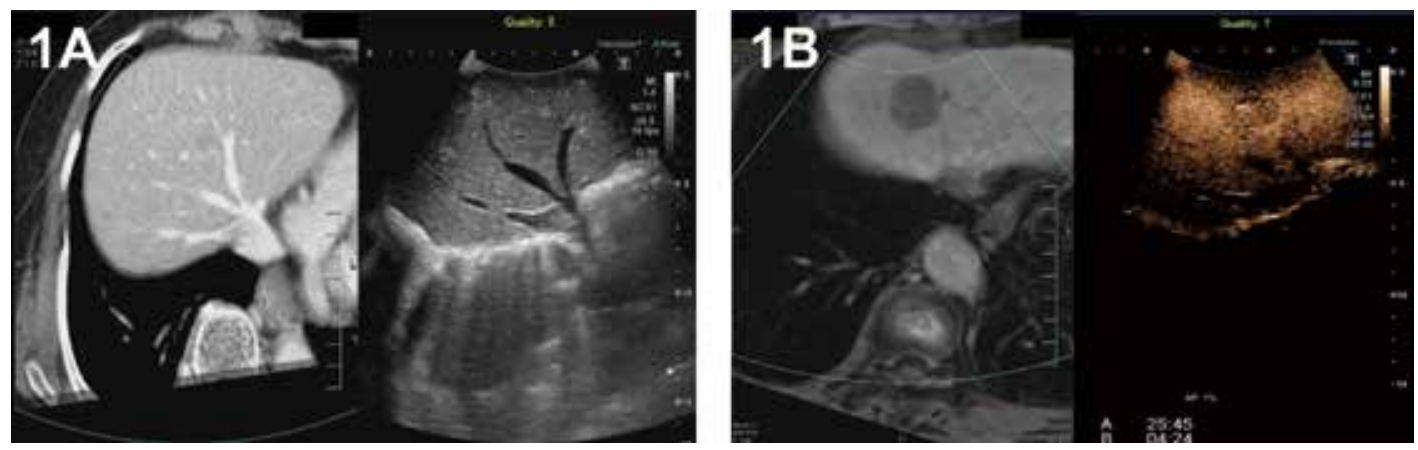

Figure 1. Image references of the RVS system. A: RVS image adjusting spatial positions of both ultrasound and CT image. The left side of the monitor indicates a CT image and the right side of the monitor indicates an ultrasound image. B: RVS image using Sonazoid enhancement for the liver tumor located on segment 8 . The left side of the monitor indicates a fusion imaging of the hepatobiliary phase in EOB-MRI, and right side of the monitor indicates Sonazoid enhancement. RVS, real-time virtual sonography; CT, computed tomography; EOB-MRI, gadolinium-ethoxybenzyl-diethylene-triaminepentaacetic acid-enhanced magnetic resonance image.

pathological findings were revealed in the post-chemotherapy state, shown as large fat droplet $(n=2)$, and sinusoidal obstruction $(\mathrm{n}=1)$.

The locations of target lesions were segment $3,5,6,7$, and 8 , respectively (Table 1). Depth of tumors from the liver surface ranged from 0.5 to $43.5 \mathrm{~mm}$. The maximum depth of detected lesions was $43.5 \mathrm{~mm}$, which indicates that this method has an advantage for detecting deep lesions (Fig. 2A, 2B). The minimum size of a liver lesion that could be detected with RVS with Sonazoid enhancement was $3.0 \mathrm{~mm}$ in diameter in Case No.6. The usefulness of the system was limited in cases where the lesion existed in the subphrenic portion (segment 7 and 8) near the root of the hepatic veins into the inferior vena cava, because the ultrasound probe could not be inserted into this space. However, some lesions located in segment 7 and 8 could be detected with RVS-CEUS (Fig. 2C, 2D). There were no severe postoperative complications (Clavien-Dindo classification : grade III or more)
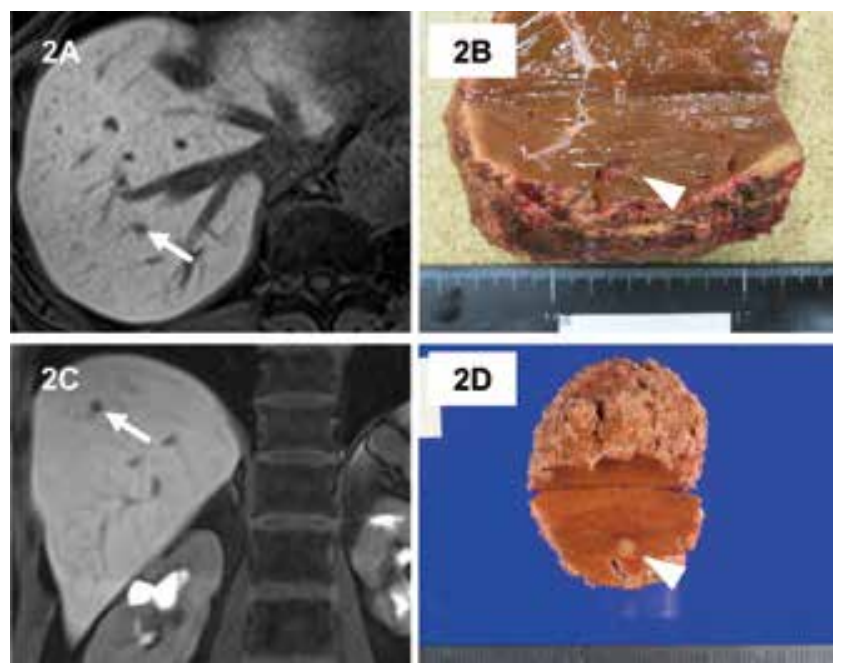

Figure 2. A : A liver lesion of segment 7 detected in the hepatobiliary phase by EOB-MRI in case No.1 (tumor No.1). The tumor size was $5.0 \mathrm{~mm}$ in diameter (arrow). B: Macroscopic finding of surgical specimen of the liver tumor (arrowhead). C : A liver lesion of segment 7 detected in the hepatobiliary phase by EOB-MRI in case No.5 (tumor No.9). The tumor size was $6.2 \mathrm{~mm}$ in diameter (arrow). D : Macroscopic finding of surgical specimen of the liver tumor (arrowhead) EOB-MRI, gadolinium-ethoxybenzyl-diethylene-triaminepentaacetic acid-enhanced magnetic resonance image. or any mortality in the patients.

Ten of eleven tumors scheduled for resection were successfully identified by this system; the detection rate was $90 \%$ (Table 2). Of these, one liver tumor located in S7 (case No.4, tumor No.7) was difficult to visualize clearly with the RVS system. The tumor was located in the dorsal part of segment 7 ; hence, the convex probe of RVS was difficult to insert into the dorsal space of the right lobe and attach to the liver surface on the posterior side. Detectability of each modality shown in Table 2 , revealed the superiority of EOB-MRI (100\%) to contrast-enhanced MDCT (50\%).

Table 2. Detectability of imaging modalities for liver metastatic tumors

\begin{tabular}{|c|c|c|c|}
\hline Modality & CE-CT & EOB-MRI & RVS-CEUS \\
\hline Detectability, number (\%) & $5 / 10(50 \%)$ & $10 / 10(100 \%)$ & $9 / 10(90 \%)$ \\
\hline
\end{tabular}

CE-CT, contrast-enhanced computed tomography ; EOB-MRI, gadolinium-ethoxybenzyl-diethylene-triaminepentaacetic acid-enhanced magnetic resonance image ; RVS-CEUS, contrast-enhanced ultrasonography with real-time virtual sonography

\section{DISCUSSION}

In this article, we described a novel technique using RVS with Sonazoid enhancement for the detection of small liver lesions, which are less than $10 \mathrm{~mm}$ in size, diagnosed by preoperative imaging such as multidetector CT or EOB-MRI. In the era of EOB-MRI and CEUS, small liver lesions downsized a few millimeters after chemotherapy are often detected with these new modalities preoperatively. Therefore, hepatobiliary surgeons sometimes recognize these small lesions for hepatic resection during surgery, and sometimes find it very difficult to detect them intraoperatively with the existing traditional modalities such as the normal ultrasound. Arita et al. reported the usefulness of IO-CEUS in identifying disappearing liver metastases from colorectal cancer after chemotherapy (15). For intraoperative diagnosis of liver lesions, IO-CEUS with Sonazoid enhancement has the highest detection rate (sensitivity) and may be the most variable modality. This RVS with CEUS method can add to the position or recognition diagnostic ability of IO-CEUS. To our knowledge, this is a novel report about intraoperative RVS using CEUS (Sonazoid enhancement) for hepatic resection. 
Previously, very few technical papers about RVS for hepatic resection were reported. Kasuya et al. described that RVS with indocyanine green (ICG) fluorescence navigation was useful for resecting US invisible liver tumors (16). ICG fluorescence navigation is an excellent surgical navigation method utilizing the rapid binding of ICG to plasma protein in vivo and the incorporation of most ICG into hepatic parenchymal cells. However, the detection of liver tumors with ICG fluorescence imaging could be indicated for only superficial liver lesions, because the excitation light of ICG fluorescence does not reach the deep layer of the liver. In this study, we could detect a tumor of 43.5 $\mathrm{mm}$ maximum depth from the liver surface; that depth is only dependent on the ability of the ultrasound system with Sonazoid enhancement. Satou et al. reported the usefulness of an RVS system combined with preoperative $3 \mathrm{D}$ simulation images to navigate the detection of tumors and for navigation of the resection plane for hepatic resection (17). This system may be very useful for intraoperative tumor detection and recognition of liver anatomy in hepatic resection. Since this system was not used with CEUS technique such as RVS with Sonazoid enhancement, small isoechoic tumors may not be visualized on the ultrasound monitor as a real-time reference image. We believe that the ultrasound reference image on the monitor should visualize the target tumor for precise guiding of transection plane, which leads to radical resection. From the advantage of our RVS with CEUS method and the result of this study, when the target liver lesion is very small with a size less than $10 \mathrm{~mm}$, we prefer this RVS system with CEUS for the surgery.

This method has a few limitations. In cases of the lesion located in the subphrenic, posterior, or caudate segments, the ultrasound probe could not be inserted into the space, because the convex type probe is only available for RVS technology at present. In such cases where the lesion is located in these segments, it is difficult to reconstruct the fusion image on the monitor. Additionally, the lesion located in very right-posterior side need to mobilize the right lobe of the liver to attach the ultrasound probe. During liver mobilization, the liver is transformed to a different form from the preoperative imaging. Therefore, it is too difficult to merge the ultrasound images of liver mobilization and CT/MRI images together in RVS system. In the future, it is expected that a smaller ultrasound probe compatible with both RVS and MBs enhancement would be developed, and this probe would make it possible to use this method for difficult situations.

Recently, several intraoperative navigation technologies for liver surgery have been reported, e.g. 3D printed liver models, augmented reality by projection mapping technology, and so on. The usefulness of $3 \mathrm{D}$ printed synthetic liver has been reported in liver surgery fields such as hepatic resection, liver transplantation, and resection perihilar cholangiocarcinoma (18-21). Having the physical model during operations provides greater intuitive navigation for critical areas, and this helps to overcome difficult orientation because these models can be physically positioned in the most desired fashion (19). Igami et al. reported that 3D printed liver could be useful in hepatic resection for small tumors that are invisible by intraoperative ultrasonography (18). They described that there are some disadvantages of 3D printing technology, such as requirement of long time to print $3 \mathrm{D}$ liver models and high cost of production. Augmented reality with projection mapping technology could also be a promising navigation tool for the detection of small tumors in liver surgery. Ntourakis et al. reported that augmented reality might be a helpful navigation tool for the resection of small liver metastases that have been shrunk by preoperative chemotherapy (22). These technologies are estimated with virtual reconstruction from preoperative images, so surgeons should consider accuracy and that errors of a few millimeters may occur during virtual reconstruction. Our strategy of this study is presented tumor detection by the real existence diagnosis using Sonazoid-enhancing ultrasound. Therefore, this method may minimize the error between visualization with RVS system and real positioning of the target tumor. Moreover, the combined use of these navigation technologies would make it easier to detect small or missing liver tumors. To our knowledge, the laparoscopic system for RVS is not yet available for commercial use. Recently, laparoscopic liver resections have been widely applied for liver metastases. The laparoscopic system for RVSCEUS could be extremely useful for the detection of tumors when performing this surgery, since laparoscopic liver resection is limited to manipulating instruments and surgical space rather than being an open liver resection.

In conclusion, this study indicated that intraoperative RVS with Sonazoid enhancement was useful for detecting small metastatic liver lesions after chemotherapy and is a viable option for hepatic resection of a size less than $10 \mathrm{~mm}$ in diameter.

\section{ACKNOWLEDGEMENT}

None.

\section{DISCLOSURE}

There were no conflicts of interest or financial support.

\section{REFERENCES}

1. Kopetz S, Chang GJ, Overman MJ, Eng C, Sargent DJ, Larson DW, Grothey A, Vauthey JN, Nagorney DM, McWilliams RR : Improved survival in metastatic colorectal cancer is associated with adoption of hepatic resection and improved chemotherapy. J Clin Oncol 27 : 3677-3683, 2009.

2. Folprecht G, Grothey A, Alberts S, Raab HR, Kohne $\mathrm{CH}$ : Neoadjuvant treatment of unresectable colorectal liver metastases : correlation between tumour response and resection rates. Ann Oncol $16:$ 1311-1319, 2005.

3. Folprecht G, Gruenberger T, Bechstein WO, Raab HR, Lordick F, Hartmann JT, Lang H, Frilling A, Stoehlmacher J, Weitz J, Konopke R, Stroszczynski C, Liersch T, Ockert D, Herrmann T, Goekkurt E, Parisi F, Köhne CH : Tumour response and secondary resectability of colorectal liver metastases following neoadjuvant chemotherapy with cetuximab : the CELIM randomised phase 2 trial. Lancet Oncol $11: 38-47,2010$.

4. Auer RC, White RR, Kemeny NE, Schwartz LH, Shia J, Blumgart LH, Dematteo RP, Fong Y, Jarnagin WR, D'Angelica MI : Predictors of a true complete response among disappearing liver metastases from colorectal cancer after chemotherapy. Cancer $116: 1502-1509,2010$.

5. Tanaka K, Takakura H, Takeda K, Matsuo K, Nagano $\mathrm{Y}$, Endo I : Importance of complete pathologic response to prehepatectomy chemotherapy in treating colorectal cancer metastases. Ann Surg 250 : 935-942, 2009.

6. van Vledder MG, de Jong MC, Pawlik TM, Schulick RD, Diaz LA, Choti MA : Disappearing colorectal liver metastases after chemotherapy : should we be concerned? J Gastrointest Surg $14: 1691-1700,2010$.

7. Kuhlmann K, van Hilst J, Fisher S, Poston G : Management of disappearing colorectal liver metastases. Eur J Surg Oncol 42 : 1798-1805, 2016.

8. Salvatore V, Borghi A, Piscaglia F : Contrast-enhanced ultrasound for liver imaging : recent advances. Curr Pharm 
Des 18: 2236-2252, 2012.

9. Schuhmann-Giampieri G, Schmitt-Willich H, Press WR, Negishi C, Weinmann HJ, Speck U : Preclinical evaluation of Gd-EOB-DTPA as a contrast agent in MR imaging of the hepatobiliary system. Radiology 183: 59-64, 1992.

10. Bluemke DA, Sahani D, Amendola M, Balzer T, Breuer J, Brown JJ, Casalino DD, Davis PL, Francis IR, Krinsky G, Lee FT Jr, Lu D, Paulson EK, Schwartz LH, Siegelman ES, Small WC, Weber TM, Welber A, Shamsi K : Efficacy and safety of MR imaging with liver-specific contrast agent: U.S. multicenter phase III study. Radiology 237 : 89 98, 2005.

11. Kudo M : Will Gd-EOB-MRI change the diagnostic algorithm in hepatocellular carcinoma? Oncology $78: 87-93$, 2010.

12. Kudo M : New sonographic techniques for the diagnosis and treatment of hepatocellular carcinoma. Hepatol Res 37 : S193-S199, 2007.

13. Miyagawa T, Ishikawa S, Kimura T, Suetomi T, Tsutsumi M, Irie T, Kondoh M, Mitake T: Real-time Virtual Sonography for navigation during targeted prostate biopsy using magnetic resonance imaging data. Int J Urol 17: 855-860, 2010.

14. Nakano S, Kousaka J, Fujii K, Yorozuya K, Yoshida M, Mouri Y, Akizuki M, Tetsuka R, Ando T, Fukutomi T, Oshima Y, Kimura J, Ishiguchi T, Arai O : Impact of real-time virtual sonography, a coordinated sonography and MRI system that uses an image fusion technique, on the sonographic evaluation of MRI-detected lesions of the breast in second-look sonography. Breast Cancer Res Treat $134: 1179-1188,2012$.

15. Arita J, Ono Y, Takahashi M, Inoue Y, Takahashi Y, Saiura A : Usefulness of contrast-enhanced intraoperative ultrasound in identifying disappearing liver metastases from colorectal carcinoma after chemotherapy. Ann Surg Oncol

\section{1 : S390-S397, 2014.}

16. Kasuya K, Sugimoto K, Kyo B, Nagakawa Y, Ikeda T, Mori Y, Wada T, Suzuki M, Nagai T, Itoi T, Shimazu M, Aoki T, Tsuchida A : Ultrasonography-guided hepatic tumor resection using a real-time virtual sonography with indocyanine green navigation (with videos). J Hepatobiliary-Pancreat Sci 18: 380-385, 2011.

17. Satou S, Aoki T, Kaneko J, Sakamoto Y, Hasegawa K, Sugawara Y, Arai O, Mitake T, Miura K, Kokudo N : Initial experience of intraoperative three-dimensional navigation for liver resection using real-time virtual sonography. Surgery $155: 255-262,2014$.

18. Igami T, Nakamura Y, Hirose T, Ebata T, Yokoyama Y, Sugawara G, Mizuno T, Mori K, Nagino M : Application of a three-dimensional print of a liver in hepatectomy for small tumors invisible by intraoperative ultrasonography : preliminary experience. World J Surg 38 : 3163-3166, 2014.

19. Zein NN, Hanouneh IA, Bishop PD, Samaan M, Eghtesad B, Quintini C, Miller C, Yerian L, Klatte R: Three-dimensional print of a liver for preoperative planning in living donor liver transplantation. Liver Transpl 19:1304-1310, 2013.

20. Soejima Y, Taguchi T, Sugimoto M, Hayashida M, Yoshizumi T, Ikegami T, Uchiyama H, Shirabe K, Maehara $\mathrm{Y}$ : Three-dimensional printing and biotexture modeling for preoperative simulation in living donor liver transplantation for small infants. Liver Transpl 22 : 1610-1614, 2016.

21. Takagi K, Nanashima A, Abo T, Arai J, Matsuo N, Fukuda T, Nagayasu T: Three-dimensional printing model of liver for operative simulation in perihilar cholangiocarcinoma. Hepatogastroenterology 61 : 2315-2316, 2014.

22. Ntourakis D, Memeo R, Soler L, Marescaux J, Mutter D, Pessaux P : Augmented Reality Guidance for the Resection of Missing Colorectal Liver Metastases : An Initial Experience. World J Surg 40 : 419-426, 2016. 